individual formulations. Thus there is a fundamental inconsistency (however small) between the opacities and equation of state quantities that are essential for pulsation analysis.

This problem could be removed if the opacity groups could provide the equation of state data that they used in construction of the opacity tables. Similarly, separate tables with opacity derivatives would give far more stable and self-consistent pulsation results if those derivatives are provided as primary quantities and not as derived by numerical differentiation from the supplied tables.

\title{
LIST OF POSTER PAPERS:
}

1. Atomic Data for Bound-Bound and Bound-Free Opacities of Hydrogen in a Strong Magnetic Field

N. Merani, J. Main, G. Wunner (Lehrstuhl fur Theoretische Physik I, Ruhr-Universitat Bochum)

2. Influence of Nonideal Effects of Dense Plasma on Solar Interior Opacity

G. Luo (Yunnan Observatory, Chinese Academy of Sciences, Kunming, P.R. China)

3. Low Temperature Rosseland Opacities

D.R. Alexander (Physics Department, Wichita State University, Wichita, KS 67260, U.S.A.)

4. Stellar Opacities for Very Low Mass Stars and Brown Dwarfs

Takashi Tsuji and Keiichi Ohnaka (Institute of Astronomy, The University of Tokyo, Mitaka, Tokyo, 181 Japan)

5. Low-Temperature Opacities Applied to Solar Calibrated Models

C. Neuforge (Institut d'Astrophysique de l'Universite de Liege, 5 Avenue de Cointe, B-4000 Liege,

Belguim), J. Provost, G. Berthomieu, P. Morel (Department Cassini, Observatoire de la Cote d'Azur, BP 229, 06304 Nice Cedex 4, France)

6. Atomic Structure Calculations and New Los Alamos Astrophysical Opacities N.H. Magee, J. Abdallah, Jr., R.E.H. Clark, J.S. Cohen, L.A. Collins, G. Csanak, C.J. Fontes, A. Gauger, J.J. Keady, D.P. Kilcrease and A.L. Merts (Theoretical Division, Los Alamos National Laboratory)

7. Critically Evaluated Data and Databases on Atomic Spectra-Energy Levels, Wavelengths, Transition Probabilities, Shape Parameters

J. Fuhr, D. Kelleher, W.C. Martin, A. Musgrove, J. Sugar and W.L. Wiese (National Institute of Standards and Technology (NIST), Gaithersburg, MD 20899, USA)

8. Analytic Fits for the Partial Photoionization Cross-Sections

D.G. Yakovlev (Ioffe Institute of Physics and Technology, St. Petersburg, Russia) and D.A. Verner (Kapteyn Astronomical Institute, Groningen, The Netherlands)

9. Atomic Data for Absorption Lines from the Ground Level at Wavelengths Longward of $228 \AA$ D.A. Verner and P.D. Barthel (Kapteyn Astronomical Institute, Groningen, The Netherlands) and 
David Tytler (University of California, Dan Diego, USA)

10. Theoretical Atomic Parameters for Databases

Z. Rudzikas, P. Bogdanovich (State Institute of Theoretical Physics and Astronomy, A. Gostauto 12, 2600, Vilnius, Lithuania)

11. Lines of Ga II and Ga III in the Ultraviolet Spectrum of Peculiar B-type Stars

Frorella Castelli (CNR-GNA and Osservatorio Astronomico, Via G.B. Tiepolo 11, 34131

Trieste, Italy) and M. Parthasarathy (Indian Institute of Astrophysics, Bangalore 560034, India)

12. Oscillator Strengths for $\mathrm{Zr}$ I and $\mathrm{Zr}$ II and the Solar Abundance of Zirconium

P. Bogdanovich, Z. Rudzikas and G. Tautvai (State Institute of Theoretical Physics and Astronomy, Gostauto 12, 2600, Vilnius, Lithuania)

13. "Forbidden" Transitions in $5 \mathrm{p}^{\mathrm{k}}(\mathrm{k}=1-5)$ Configurations

E. Biemont (Research Director of the Belgian FNRS, Institut d'Astrophysique, Universite de Liege, 5, Avenue de Cointe, B-4000 Liege, Belgium), J.E. Hansen (Van der Waals-Zeeman Laboratorium, Universiteit van Amsterdam, NL-1018 XE Amsterdam, The Netherlands) and C.J. Zeippen (Observatoire de Paris, Section d'Astrophysique de Meudon, URA 173 et DAEC, F-92190 Meudon, France)

14. The Equation of State in the Chemical Picture: A Grand Canonical Approach

G. Luo (Yunnan Observatory, Chinese Academy of Sciences, Kunming, P.R. China)

15. On the Stark Broadening of NaIX Spectral Lines

Milan S. Dimitrijevic (Astronomical Observator, Volgina 7, 11050 Beograd, Yugoslavia) and Sylvie Sahal-Brechot (Observatoire de Paris, 92195 Meudon, Cedex, France)

16. Stark Broadening of AI XI Spectral Lines

Milan S. Dimitrijevic (Astronomical Observatory, Volgina 7, 11050 Beograd, Yugoslavia) and Sylvie Sahal-Brechot (Observatoire de Paris, 92195 Meudon, Cedex, France)

17. The Modified Semiempirical Approach for Complex Ion Lines of Astrophysical Interest L.C. Popovic and M.S. Dimitrijevic (Astronomical Observatory, Volgina 7, 11050 Belgrade, Yugoslavia)

18. Why Stark Broadening Parameters are Important for Opacity Calculations of Stellar Atmospheres Alain Lesage (DASGAL Observatoire de Paris, Section d'Astrophysique de Meudon, 92195 Meudon, France)

19. Radiative Accelerations in Stars Using OPACITY Project Data

G. Alecian (Laboratoire d'Astrophysique Extragalactique et de Cosmologie - URA173 (CNRS,

Observatoire de Paris, Universite de Paris VI, DAEC, Observatorie de Meudon, F-92195 Meudon

Cedex, France) and J. Tully (Laboratoire G.D. Cassini, CNRS-URA1362, Observatoire de la Cote d'Asur, BP 229, F-06304 Nice Cedex 4, France) 
20. Stellar Winds in A Type Supergiants

Eva Verdugo and Antonio Talavera (IUE Observatory, European Space Agency, P.O. Box 50727, 28080 Madrid, Spain)

21. The Non LTE Analysis of Procyon on the Basis of the New Kunucz's Model Atmosphere L.I. Mashonkina, N.N. Shimanskaya, and V.V. Shimansky (Kazan State University)

22. Spherically Symmetric NLTE Model Atmospheres Using Approximate Lambda Operators Jini Kubat (Astronomical Institute, Academy of Sciences of the Czech Republic, 25165 Ondrejov, Czech Republic)

23. First Combined Stellar Structure and Atmosphere Models: Interior and Spectral Evolution of Massive Stars

D. Schaerer (Geneva Observatory, Switzerland), A. de Koter (NASA/GSFX, USA), W. Schmutz (Institut fur Astronomie, ETH Zurich, Switzerland), A. Maeder (Geneva Observatory)

24. The Vertical Oscillations of Thin Accretion Discs with the OPAL Opacities R. Kanetake, M. Takeuti (Astronomical Institute, Tohoku University, Sendai 980 Japan) and J. Fukue (Astronomical Institute, Osaka Kyoiku University, Kasihara, Osaka 582 Japan)

25. Hydrodynamic Simulation of a Double-Mode Cepheid with the OPAL Opacities M. Takeuti (Astronomical Institute, Tohoku University, Sendai 980, Japan), T. Ishida (Nishi-Harima Astronomical Oservatory, Ohnadesan, Sayo-cho, Hyogo 697-53, Japan), and R. Takano (Faculty of Education, Fukushima University, Fukushima, Japan)

26. Period-Luminosity Relations for the RR Lyrae Stars in $\varphi$ Centauri

J.O. Petersen (Niels Bohr Institute for Astronomy, Physics and Geophysics, Astronomical Observatory, Copenhagen)

27. BV Aquarii: A Double-Mode RR Lyrae Variable with Peculiar Period Ratio Mikolaj Jerzykiewicz (Wroclaw University Observatory, Kopernika 11, 51-622 Wroclaw, Poland)

28. New Los Alamos Opacities and Solar Oscillations Arthur N. Cox, Robert E.H. Clark, Norman H. Magee, and Joyce A. Guzik (Los Alamos Astrophysics)

29. Abundances of Elements in the Star Gamma Draconis David F. Gray (Department of Astronomy, University of Western Ontario, Physics and Astronomy Building. London, Ontario, N64 3K7, Canada), K. Sinha and B.B. Sanwal (U.P. State Observatoru, Manora Peak, Naini Tal-263 129, India)

30. A Spectral Synthesis Study of the Magnetic CP Star HD 204411

Hulya Caliskan (Department of Astronomy, Istanbul University, 34452 University, Istanbul, Turkey), Saul J. Adelman (Department of Physics, The Citadel, Charleston, SC 29409, USA)

31. Astrophysical $g f$ values from High $S / N$ Data of A Type Stars 
Saul J. Adelman (Department odf Physics, The Citadeh, Charleston, SC 29409 USA), Austin F. Gulliver (Department of Physics and Astronomy, Brandon University, Brandon, MB, R7A $6 A 9$ Canada) and Graham Hill (Dominion Astrophysical Observatory, 5071 W. Saanich Road, Victoria, $B C$, V8X 5M6 Canada)

32. A New Class of Analytic Models for White Dwarfs Vasile Ureche (University of Cluj-Napoca, Faculty of Mathematics and Computer Science, Str. M.Kogalniceanu 1, 3400 Cluj-Napoca, Romania) and Vasile Mioc (Astronomical Institute of the Romanian Academy, Astronomical Observatory Cluj-Napoca, Str. Ciresilor 19, 3400 Cluj-Napoca, Romania)

33. Effects of Differential Rotation and Tidal Distortion on the Eigenfrequencies of Polytropic Models of Stars

C. Mohan, V.P. Singh and A.K. Lal (Dept. of Mathematics, University of Roorkee, Roorkee (U.P.), India)

34. Light Curves of $\Delta m$ 's Flare Stars with $\mathrm{Li}$ in the Pleiades

Enrique Chavira (Instituto Nacional de Astrofisica, Opitca y Electronica)

35. Calculated Late-Time Spectra of Type Ia Supernovae: Atomic Needs and Astrophysical Interest

P. Ruiz-Lapuente (Dpto. de Astronomia y Meterologia, Facultad de Fisica, Diagonal 647, 08028 Barcelona, Spain)

36. New Stellar Atomic Databases and the Ages of Globular Clusters

I. Mazzitelli, V. Caloi (Istituto di Astrofisica Spaziale CNR, CP 67, I-00044 Frascati) and F.

d'Antona (Osservatorio Astronomico di Roma, I-00040 Monte Porzio)

37. A Photoionisation Computations Database

E.Y. Vilkoviskij, S.M. Efimov and I.N. Nosov (Fessenkov Astrophysical Institute of NAS of Kazakhstan) 